\title{
Weakening Cardinality Constraints Creates Harder Satisfiability Benchmarks
}

\author{
IVOR SPENCE, Queen's University Belfast
}

\begin{abstract}
For some time, the satisfiability formulae that have been the most difficult to solve for their size have been crafted to be unsatisfiable by the use of cardinality constraints. Recent solvers have introduced explicit checking of such constraints, rendering previously difficult formulae trivial to solve. A family of unsatisfiable formulae is described that is derived from the sgen4 family but cannot be solved using cardinality constraints detection and reasoning alone. These formulae were found to be the most difficult during the SAT2014 competition by a significant margin and include the shortest unsolved benchmark in the competition, sgen61200-5-1.cnf.
\end{abstract}

Categories and Subject Descriptors: F.2.2 [Nonnumerical Algorithms and Problems]: Complexity of Proof Procedures

General Terms: Satisfiability, Benchmarks

Additional Key Words and Phrases: SAT-solvers, small hard benchmarks

\section{ACM Reference Format:}

Ivor Spence. 2015. Weakening cardinality constraints creates harder satisfiability benchmarks. ACM J. Exp. Algor. 20, 1, Article 1.4 (May 2015), 14 pages.

DOI: http://dx.doi.org/10.1145/2746239

\section{INTRODUCTION}

The boolean satisfiability problem is to determine whether it is possible to assign truth values to the Boolean variables in a propositional expression (formula) in such a way that the overall expression has the value true. The theoretical and practical importance of this problem has generated much work in developing effective solvers; every year an international competition [Järvisalo et al. 2012] is held in conjunction with the annual conference on the Theory and Applications of Satisfiability Testing. ${ }^{1}$ There are frequently significant improvements in solver technology from one competition to the next, and there are formulae with millions of literals that can be solved within the allowed CPU time, which was 5,000s in 2014.

However, this decision problem is NP-Complete [Cook 1971], inspiring the search for small difficult formulae that the best solvers find exponentially hard. We focus in this article on formulae with fewer than 1,500 literals that were not solved in the 2014 competition. The most difficult formulae for their size have always been unsatisfiable and to date have relied on cardinality constraints to ensure that they cannot be satisfied. For example, the formulae in the pigeonhole series [Haken 1984]

\footnotetext{
${ }^{1}$ http://www.satisfiability.org/.
}

Author's address: I. Spence, School of Electronics, Electrical Engineering and Computer Science, Queen's University Belfast, University Road, Belfast BT7 1NN United Kingdom; email: i.spence@qub.ac.uk. Permission to make digital or hard copies of part or all of this work for personal or classroom use is granted without fee provided that copies are not made or distributed for profit or commercial advantage and that copies show this notice on the first page or initial screen of a display along with the full citation. Copyrights for components of this work owned by others than ACM must be honored. Abstracting with credit is permitted. To copy otherwise, to republish, to post on servers, to redistribute to lists, or to use any component of this work in other works requires prior specific permission and/or a fee. Permissions may be requested from Publications Dept., ACM, Inc., 2 Penn Plaza, Suite 701, New York, NY 10121-0701 USA, fax +1 (212) 869-0481, or permissions@acm.org.

(c) 2015 ACM 1084-6654/2015/05-ART1.4 $\$ 15.00$

DOI: http://dx.doi.org/10.1145/2746239 
encode the allocation of $n$ pigeons into $n-1$ pigeonholes with no more than one pigeon per hole, which is, of course, impossible. Related series include Hirsch's hgen [Hirsch 2002], and in recent years (including 2011) the most difficult formulae for their size at the SAT competitions [Järvisalo et al. 2012] have come from the sgen [Spence 2010] family of generators.

Some solver developers [Ostrowski et al. 2002; Li 2000; Warners and Maaren 1998] have targeted particular classes of formula that has enabled very efficient solving of formulae previously found to be difficult. In particular, analysis using cardinality constraints [Biere et al. 2014] means that solvers that can solve instances of these series up to 1,200 literals in less than 1s are now available. Motivated by this, the approach that was used in the sgen4 benchmark generator has been modified so that cardinality constraints alone are not sufficient to solve the generated formulae.

The main contributions of this article are:

-A demonstration that the satisfiability instances that were previously the most difficult are not any more and an explanation of why this is the case

-A description of a new approach for generating unsatisfiable formulae with an explanation of why these should be more difficult

- The presentation of results from the SAT 2014 competition and further experiments indicating that the new formulae are now by a significant margin the most difficult known, and suggesting that they are exponentially difficult for even the best solvers

We first describe briefly the satisfiability problem and the structure of the DIMACS standard input format. We explain the reasons for trying to create difficult instances, showing how instances previously found to be difficult were constructed. After explaining how recent solvers are able to solve such instances easily, we introduce our new family of formulae. We define our measure of difficulty and present results from the 2014 SAT Competition indicating the success of this approach. Finally, we present more detailed experimental results that suggest the exponential times required by the best solvers for these benchmarks.

\section{THE SATISFIABILITY PROBLEM}

The satisfiability problem is to take a Boolean proposition (formula) in conjunctive normal form [Whitesitt 1995] and determine whether there is an assignment of the values true and false to the variables such that the whole proposition has the value true. If this is possible, then the formula is said to be satisfiable and the corresponding values of the variables constitute a satisfying model; otherwise, the formula is said to be unsatisfiable. The typical mathematical notation uses $p$ to denote a variable, $\bar{p}$ to denote the negation of $p$, and $\wedge, \vee$ to denote logical and and or, respectively. $p$ and $\bar{p}$ are known as literals. A clause is a sequence of literals separated by $\vee$ operators and enclosed in parentheses. A formula is a sequence of clauses separated by $\wedge$ operators. A formula that is used to test the performance of a solver is often called a benchmark or an instance.

Thus $(p \vee q)$ is a clause, $(p \vee q) \wedge(\bar{p} \vee \bar{q})$ is a formula that is satisfiable with $\{p, \bar{q}\}$ being a satisfying model, and the formula $(p) \wedge(q) \wedge(\bar{p} \vee \bar{q})$ is unsatisfiable.

\subsection{DIMACS Input Format}

The standard for representing formulae by text files is the DIMACS format [DIMACS 1993] and files have the standard extension . cnf-this is the format used during the SAT competitions. In such a file, the variables are represented by nonzero integers, with a positive value indicating the literal $p$ and a negative value indicating $\bar{p}$. The first line of such a file is of the form

$\mathrm{p}$ cnf num-of-variables num-of-clauses 
and thereafter clauses are expressed as zero-terminated sequences of integers (conventionally each clause is on a separate line). For example, if $p$ is represented by 1 and $q$ by 2 , then the file to represent $(p \vee q) \wedge(\bar{p} \vee \bar{q})$ is

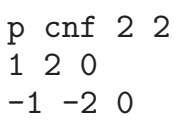

\section{CARDINALITY CONSTRAINTS}

The most effective techniques for creating small difficult formulae have to date involved partitioning variables into small groups and, within each group, generating clauses that impose constraints on the number of variables that can take a particular value. For example, at least one variable must take the value true, or at most two must take the value false [Spence 2010]. When all the constraints for a formula are considered together, they can be inconsistent (for an unsatisfiable formula) or at least only permit a small number of solutions (for a satisfiable formula), but previous solvers did not explicitly use this information.

\subsection{Unsatisfiable Cardinality Constraints}

In an unsatisfiable formula generated by sgen4, the partitions contain one group of five variables with the remaining groups containing four variables. There are clauses that guarantee that at most two variables in each group are true, meaning that overall at most (N-1)/2 variables can be true. A second and similar partition is used to guarantee that at most $(\mathrm{N}-1) / 2$ variables can be false. Taken together, it is only possible to allocate values to N-1 of the variables, thus the formula is unsatisfiable. Simulated annealing is used to create the second partition in such a way as to minimize any similarity to the first, so that, for example, variables that appear in the same group in the first partition are not in the same group in the second partition. This shuffling reduces the number of times that the same variables appear together in the same clause, which increases the difficulty of finding a solution [Spence 2010].

Formulae of this kind have until recently proved to be the most difficult for stateof-the-art solvers. It has been shown [Mikša and Nordström 2014] that they are exponentially difficult for resolution-based approaches; in 2010, a challenge was issued [Van Gelder and Spence 2010] for a particular 1,060-literal formula to be solved in less than a day. It is apparent that a formula-specific approach could have been used to recognize this structure and declare unsatisfiability quickly, but the target was not met until 2014. Solvers have now been written [Biere et al. 2014] that incorporate detection and analysis of cardinality constraints (see Section 4.2), meaning that the previously difficult formulae, including the challenge mentioned earlier, can be solved in a fraction of a second. These solvers are based on cutting planes which allow the solver to produce a short proof of unsatisfiability [Cook et al. 1987].

\subsection{Weaker Cardinality Constraints}

In satisfiable formulae generated by sgen4, weaker constraints are used so that not all possible assignments are immediately precluded. Such a formula is based on three partitions of the variables, each consisting of groups of $g$ variables (for the SAT 2013 competition a value of $g=5$ was used; see Section 4.3). Simulated annealing is again used to minimize any similarity among the partitions. The groups in the second and third partitions all intersect with the groups of the first partition in at most one variable, except for very small benchmarks, in which this is impossible to achieve. The constraints generated for the first partition guarantee that at most $p$ variables from each group are true (for the SAT 2013 competition, a value of $p=1$ was used; see Section 4.4). Those for each of the other two partitions guarantee that at least $p$ 


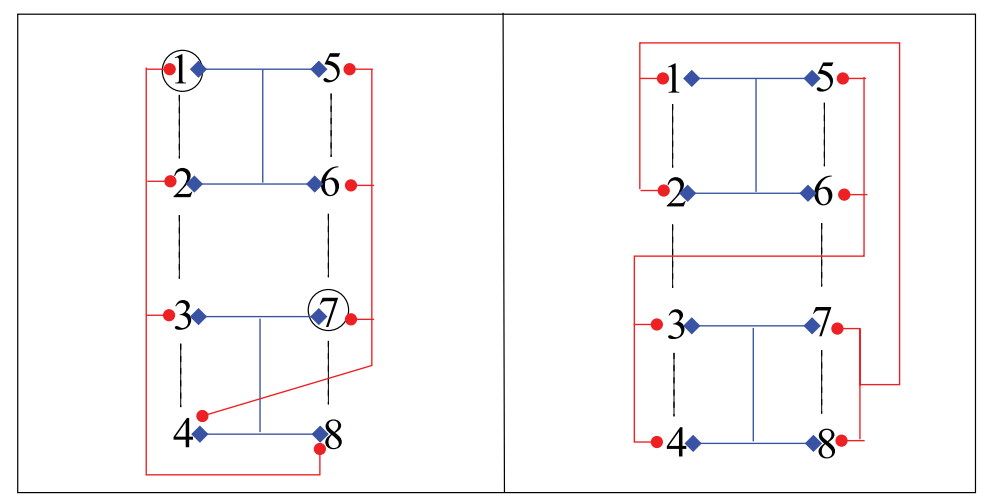

Fig. 1. Constraints corresponding to satisfiable (left) and unsatisfiable formulae.

variables from each group are true. Thus, potentially, there is a solution with exactly $n p / g$ variables being true. The question then becomes whether it is possible to identify a set of $n p / g$ variables such that, in each of the three partitions, exactly $p$ of the chosen variables occurs in each group. The sgen 4 generator generates the partitions in such a way that this is forced to be true by choosing in advance what this set of variables will be, then not shuffling them in the simulated annealing process. Analyzing cardinality constraints does not provide a quick solution to these formulae, which are among the more difficult satisfiable benchmarks, but using incomplete solvers such as sattime, formulae with more than 1,000 literals could be solved in the order of $10 \mathrm{~s}$ in 2011 . The cutting planes approach does not help here because there can be no proof of unsatisfiability for a satisfiable formula.

\section{3. sgen6: Unsatisfiability With Weaker Constraints}

Finally, we describe the improvement that has led to the sgen 6 unsatisfiable formulae that cannot be solved quickly even using cardinality constraints. In order to provide the challenge of having an unsatisfiable formula but avoid having cardinality constraints that, on their own, guarantee unsatisfiability, we investigated removing the restriction on partitioning that sgen 4 enforced. This means that satisfiability of generated formulae is not guaranteed but depends on the random partitioning process. If it is possible to choose a set of $n p / g$ variables in such a way that in every partition there are exactly $p$ such variables in each group, then the formula is satisfiable; otherwise, it is not. As the size of the formula increases, it can be seen empirically that unsatisfiability is more likely. For example, with a group size of 5 , the probability of a satisfiable formula is approximately $50 \%$ at 540 literals. Within the sgen 6 tool, an exhaustive (computationally expensive) search can be carried out on request to ensure that only unsatisfiable benchmarks are generated. These are the kinds of formula that proved to be so difficult in the 2014 competition.

Figure 1 illustrates the constraints corresponding to one satisfiable (left) and one unsatisfiable formula in which $g=4$ and $p=1$. The vertical black dashed lines represent the at-most-one constraints; it can be seen that in both formulae these constraints refer to the unshuffled partition $\{\{1,2,3,4\},\{5,6,7,8\}\}$. The solid red (with dots) and blue (with diamonds) lines represent the at-least-one constraints that are applied to shuffled partitions. For each example, choosing one positive variable from each of the two elements of the first partition, with the at-most-one constraints, means choosing a maximum of two positive variables in total. The second and third partitions both lead to two at-least-one constraints, therefore a minimum of two positive variables have to 


\begin{tabular}{|c|c|}
\hline $\begin{array}{l}\text { Satisfiable with the model } \\
\{1,-2,-3,-4,-5,-6,7,-8\}\end{array}$ & Unsatisfiable \\
\hline$p \operatorname{cnf} 816$ & $\mathrm{p} \operatorname{cnf} 816$ \\
\hline$-1-20$ First partition & $-1-20$ First partition \\
\hline$-1-30 \quad\{1,2,3,4\},\{5,6,7,8\}$ & $-1-30 \quad\{1,2,3,4\},\{5,6,7,8\}$ \\
\hline$-1-40$ "at most one" & $-1-40$ "at most one" \\
\hline$-2-30$ black dashed lines & $-2-30$ black dashed lines \\
\hline $\begin{array}{lll}-2 & -4 & 0\end{array}$ & $\begin{array}{lll}-2 & -4 & 0\end{array}$ \\
\hline $\begin{array}{lll}-3 & -4 & 0\end{array}$ & $\begin{array}{lll}-3 & -4 & 0\end{array}$ \\
\hline $\begin{array}{lll}-5 & -6 & 0\end{array}$ & $\begin{array}{lll}-5 & -6 & 0\end{array}$ \\
\hline $\begin{array}{lll}-5 & -7 & 0\end{array}$ & $\begin{array}{lll}-5 & -7 & 0\end{array}$ \\
\hline $\begin{array}{lll}-5 & -8 & 0\end{array}$ & $\begin{array}{lll}-5 & -8 & 0\end{array}$ \\
\hline $\begin{array}{lll}-6 & -7 & 0\end{array}$ & $\begin{array}{lll}-6 & -7 & 0\end{array}$ \\
\hline $\begin{array}{lll}-6 & -8 & 0\end{array}$ & $\begin{array}{lll}-6 & -8 & 0\end{array}$ \\
\hline $\begin{array}{lll}-7 & -8 & 0\end{array}$ & $\begin{array}{lll}-7 & -8 & 0\end{array}$ \\
\hline 12380 Second partition & 12780 Second partition \\
\hline $\begin{aligned} 45610 & \{1,2,3,8\},\{4,5,6,7\} \\
& \text { "at least one" } \\
& \text { red lines with circles }\end{aligned}$ & $\begin{aligned} 34560 & \{1,2,7,8\},\{3,4,5,6\} \\
& \text { "at least one" } \\
& \text { red lines with circles }\end{aligned}$ \\
\hline 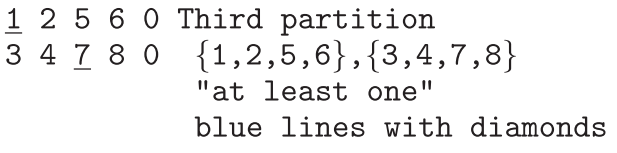 & $\begin{array}{rlllll}1 & 2 & 5 & 6 & 0 & \text { Third partition } \\
3 & 4 & 7 & 8 & 0 & \\
& & & \\
& & \\
& & & \text { blut least line lines with diamonds }\end{array}$ \\
\hline
\end{tabular}

Fig. 2. cnf files for the example of satisfiable and unsatisfiable formulae.

be chosen. Thus, overall, there must be exactly two. As can be seen, choosing 1 and 7 (circled) satisfies all the constraints in the left diagram. The right diagram, however, cannot be satisfied. For example choosing 1 means that the second positive variable must come from the column $\{5,6,7,8\}$. Choosing 5 or 6 leaves $\{3,4,7,8\}$ (blue with diamonds) unsatisfied, and choosing 7 or 8 leaves $\{3,4,5,6\}$ (red with dots) unsatisfied.

Figure 2 presents the cnf files corresponding to these two sets of constraints. The two formulae have the same structure with respect to cardinality constraints, and their satisfiability depends on the exact partitions, demonstrating that cardinality detection alone is not sufficient to solve these formulae. We have not proved the impossibility of a short proof of unsatisfiability, but no known solver has been found to take advantage of one.

\section{RESULTS}

We present published results from the SAT2014 competition, as well as results from our own experiments. The platform for SAT2014 was

-Operating System: Linux Centos 5.5

-Processor(s): 2 Hex-core Xeon 5680

-Memory: 24GB

-Timeout: $5,000 \mathrm{~s}$

For our experiments the platform was 
-Operating System: Ubuntu 13.04

-Processor(s): 2 Quad-core Xeon 5430

-Memory: 8GB

-Timeout: 30,000 s

All the programs are sequential.

\subsection{Smallest Known Time Metric}

In the absence of any absolute measure of difficulty, we introduce a technique for comparing the relative difficulty of two formulae by considering for each its size, measured by the number of literals it contains, and the shortest time to solution of any known solver applied to that formula (the execution platform is implicit). This is also known as the execution time of the Virtual Best Solver (VBS) [Xu et al. 2012]. Note that we do not insist on the same solver being used in both cases-we are looking for formulae that are resistant to solution by any solver. We define a partial order that indicates whether it is possible to compare the difficulty of two given benchmarks. Clearly, the value depends on the set of solvers used, and for any given formula, the inclusion of a new solver can decrease but cannot increase the shortest time to solution.

Suppose that $S$ is a set of solvers and $F$ is a set of formulae. Given $s \in S, f \in F$, we denote by $t(s, f)$ the execution time of the solver $s$ applied to the formula $f$. We define $\operatorname{vbs}_{S}(f)=\min _{s \in S} t(s, f)$, that is, the smallest time of any solver $s \in S$ when applied to $f$. We are interested in the difficulty of formulae in terms of their length, thus we denote the number of literals in a formula $f$ by $\operatorname{lits}(f)$.

We then define a partial order $\preceq_{S}$ on formulae that is intended to formalize the notion of one formula being less difficult than another with respect to the set of solvers $S$. If two formulae are the same size, then the one with smaller VBS execution time is said to be easier. If the formulae have the same VBS execution time, then the one containing more literals is said to be easier. If the longer of two formulae has the smaller VBS time, then it is clearly easier. Finally, if the longer formula also has a greater VBS time, then we cannot compare the difficulty of the two formulae.

Formally, the relation $f_{1} \preceq_{S} f_{2}$ indicates that if $f_{1}$ is easier, then $f_{2}$ (or equally difficult) where

$$
\begin{aligned}
& f_{1} \preceq_{S} f_{2}=\left\{\left(f_{1}, f_{2}\right) \mid \operatorname{lits}\left(f_{1}\right) \geq \operatorname{lits}\left(f_{2}\right) \wedge \operatorname{vbs}_{S}\left(f_{1}\right) \leq \operatorname{vbs}_{S}\left(f_{2}\right)\right\} \\
& f_{1}=_{S} f_{2}=\left\{\left(f_{1}, f_{2}\right) \mid \operatorname{lits}\left(f_{1}\right)=\operatorname{lits}\left(f_{2}\right) \wedge \operatorname{vbs}_{S}\left(f_{1}\right)=\operatorname{vbs}_{S}\left(f_{2}\right)\right\}
\end{aligned}
$$

For this relation to be useful, the set of solvers should be as large as possible, or should at least include the best-known solvers for the formulae in question. In this article, we use the set of solvers submitted to the SAT Competition 2014 together with a small number of others that have been found to be particularly effective on small difficult benchmarks. No other solvers are known whose inclusion would materially alter the conclusions.

Figure 3 illustrates the different possibilities when we attempt to compare two formulae. Each part of this figure represents a scatter plot of VBS time against number of literals. The first two sketches are hypothetical and the third illustrates the situation for the four sample formulae listed in Table I with data from the SAT Competition 2014. Formulae A, B, and D are unsatisfiable and formula C is satisfiable. In the first sketch, $f_{1}$ is easier than any of $f_{2}, f_{3}, f_{4}$. It is longer than $f_{2}$ for the same execution time, takes less time than $f_{4}$ for the same number of literals, and is both longer and faster than $f_{3}$. In the second sketch, $f_{1}$ is shorter than $f_{2}$ but also takes less time, thus their difficulties cannot be compared. In the third sketch, it can seen, for example, that 


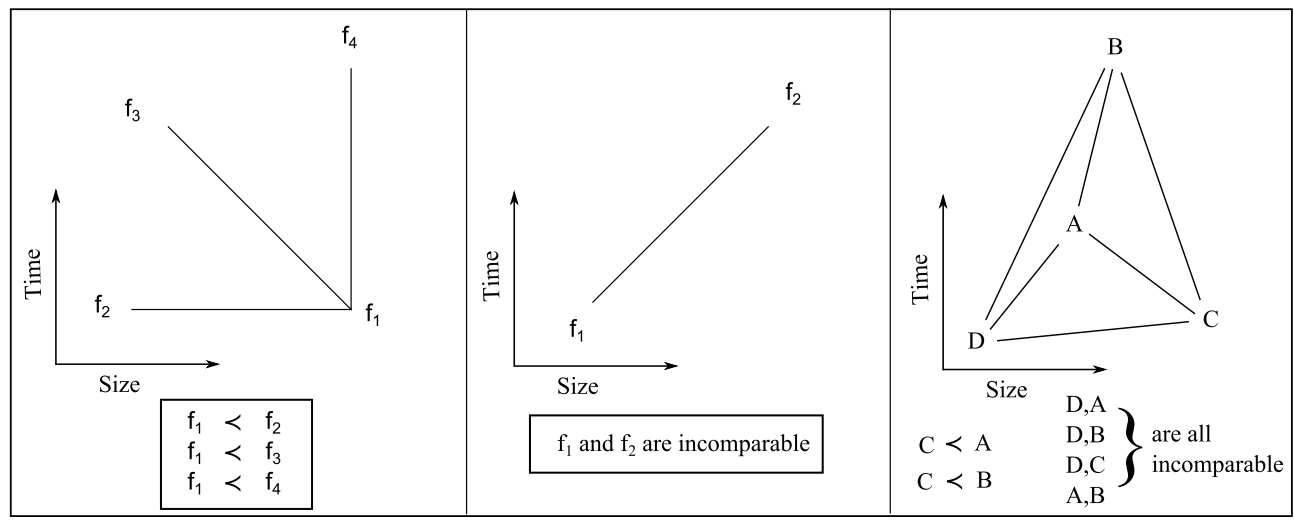

Fig. 3. Relative difficulty of two formulae.

Table I. Example Results

\begin{tabular}{l|l|c|c|c}
\hline Id & Formula & Lits & Best Solver & VBS Time(s) \\
\hline A & sgen6-840-5-1 & 840 & BFS-Glucose_mem_8_85 & 279 \\
\hline B & sgen6-960-5-1 & 960 & glueSplit_clasp & 692 \\
\hline C & sgen4-sat-220-8 & 1,320 & RSeq2014 & 11.1 \\
\hline D & edges-024-4-5667555-1-00 & 612 & Lingeling & 0.004 \\
\hline
\end{tabular}

edges-024-4-5667555-1-00 has a very short minimum execution time (0.004s), but because it is also smaller than the other formula, we cannot infer that it is easier. By contrast, since sgen4-sat-220-8 (C) is larger than sgen6-960-5-1 (A) as well as having a shorter execution time, we say that sgen4-sat-220-8 is easier than sgen6-960-5-1.

\subsection{Solver Incorporating Cardinality Detection}

First, we demonstrate that the use of a solver incorporating cardinality detection can quickly solve formulae previously found to be very difficult. sgen4 formulae were used in the SAT 2014 Competition, but pigeon-hole and hgen8 were not, thus we ran the solver sat4j-detectcards [Biere et al. 2014] on a collection of pigeon-hole, hgen8, and sgen4 formulae from 500 to 1,200 literals. Figure 4 shows the execution times for this solver for the two pigeon-hole formulae within this range and for 20 randomly generated hgen8 and sgen4 formulae for each of a range of requested numbers of literals. Note that hgen8 does not generate formula with exactly a given number of literals, hence the horizontal blurring of each cluster of values. It can be seen that, in every case, the execution time was less than 1s. By contrast, this solver takes more than 10,000 s to solve a 540 literal sgen 6 formula.

\subsection{Group Size}

We now address the issue of determining the optimum values for the size of the groups of variables $(g)$ and by the number of variables within each group that can and must be positive $(p)$. The values $g=5$ and $p=1$ had been found empirically to be the best for the satisfiable benchmarks in sgen4; it was anticipated that these values would also be best for the unsatisfiable benchmarks of sgen6. For a group of size $g$, of which $p$ variables are positive, there will be $\frac{g !}{p !(g-p) !}=\left(\begin{array}{l}g \\ p\end{array}\right)$ possible ways of assigning these positive variables. It might be anticipated that, for a given number of groups, the solver execution time will increase as $\left(\begin{array}{l}g \\ p\end{array}\right)$ increases. The experiments described in Sections 4.3 and 4.4 use the solver glueSplit_clasp. If $p$ is fixed at, for example, 1 , then $\left(\begin{array}{l}g \\ p\end{array}\right)=g$ 


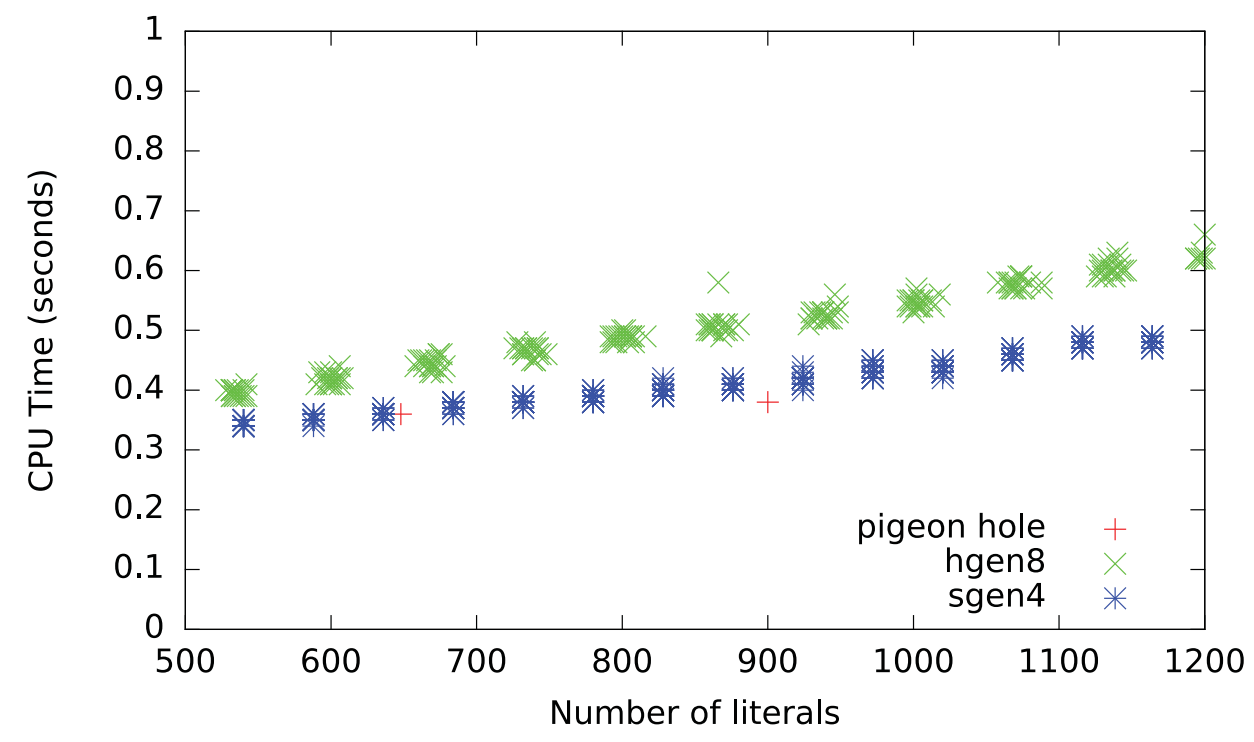

Fig. 4. Performance of sat4j-detectcards on previously difficult instances.

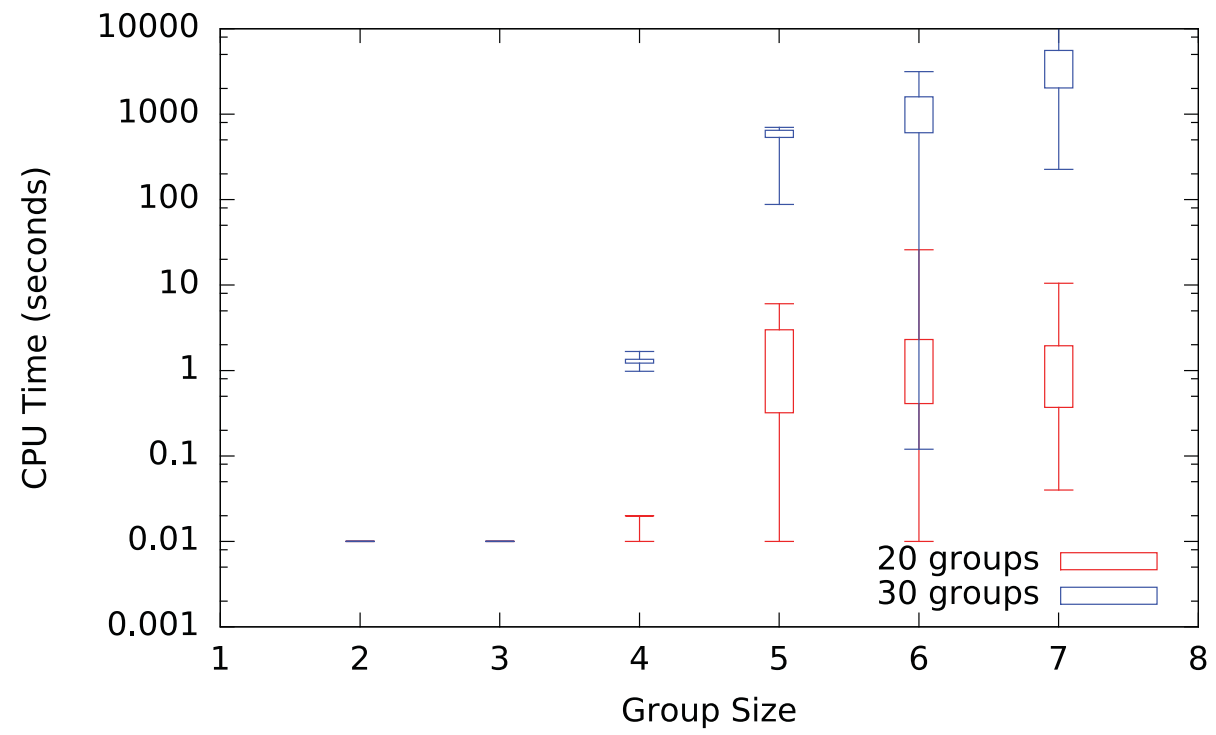

Fig. 5. Execution time against group size for a fixed number of groups.

and execution time might be expected to increase with group size. Figure 5, in which each candlestick shows the minimum, lower quartile, upper quartile, and maximum execution times, plots glueSplit_clasp against 20 sgen 6 benchmarks for each group size from 2 to 7 with 20 groups and with 30 groups. It can be seen that execution time does broadly increase with group size.

In addition to increasing the execution time for a fixed number of groups, however, increasing the value of $g$ also increases the total number of literals. Given that we are interested in execution time for a given number of literals, we must take this into consideration. Instead of fixing the number of groups, we must fix the number of literals 


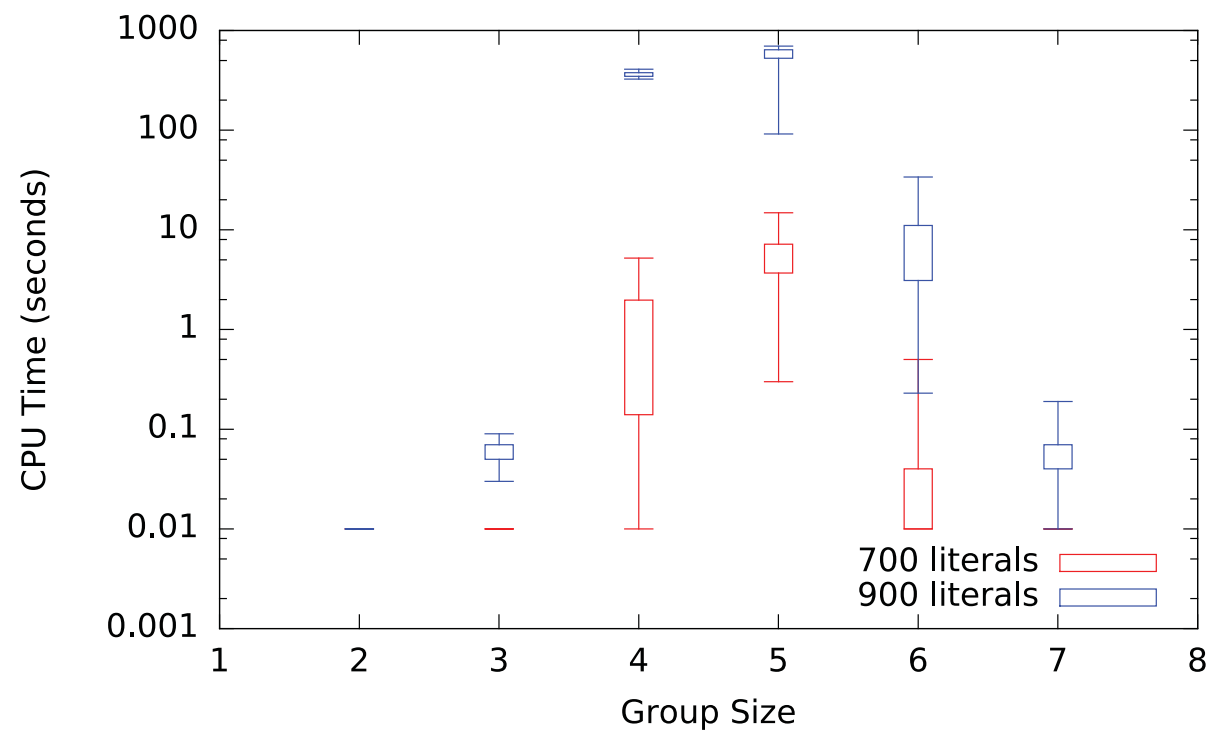

Fig. 6. Execution time against group size for a fixed number of literals.

(at least approximately). Figure 6 shows execution times for glueSplit_clasp against 20 benchmarks for each group size from 2 to 7 with limits of approximately 700 and 900 literals. Note that the number of literals is not fixed exactly because of the constraint imposed by the relationship among $g, p$, number of groups and number of literals.

It can be seen that a group size of 5 , as used previously, does provide the greatest execution times for a given number of literals. It offers the best combination of providing sufficient complexity per group without requiring too many literals per group.

\subsection{Positive Variables per Group}

If $g$ is fixed at, for example, 5 , then $\left(\begin{array}{l}g \\ 1\end{array}\right)=5,\left(\begin{array}{l}g \\ 2\end{array}\right)=10$, and $\left(\begin{array}{l}g \\ 3\end{array}\right)=10$, and execution time might be expected to increase at least as $p$ increases from 1 to 2 . Figure 7 shows the execution times for glueSplit_clasp against 20 benchmarks with $g=5$ and $p=1,2,3$ with 20 groups and with 30 groups. It can be seen in practice that, even with a fixed number of groups, the execution time does not increase with $p$. Given that the number of literals does increase with $p$, it is clear that using $p=1$ gives the best results. It should be noted that with $p>1$, it was not possible to generate any unsatisfiable benchmarks, thus the results reported are for satisfiable ones. It is possible that an incomplete solver could give even smaller execution times when $p>1$, but this would only confirm the result that $p=1$ is the best choice.

\subsection{SAT 2014 Competition}

In this section, we present the results obtained by submitting our formulae to be used as benchmarks in the SAT 2014 Competition. As in previous years, the competition was divided into three sections based on the origin of the corresponding benchmarks:

Application. These benchmarks reflect the expression of a real-world problem as a satisfiability instance. They are typically large but can sometimes be surprisingly easy for solvers.

Random. These benchmarks are randomly generated but typically with carefully controlled ratios of numbers of clauses to numbers of literals. 


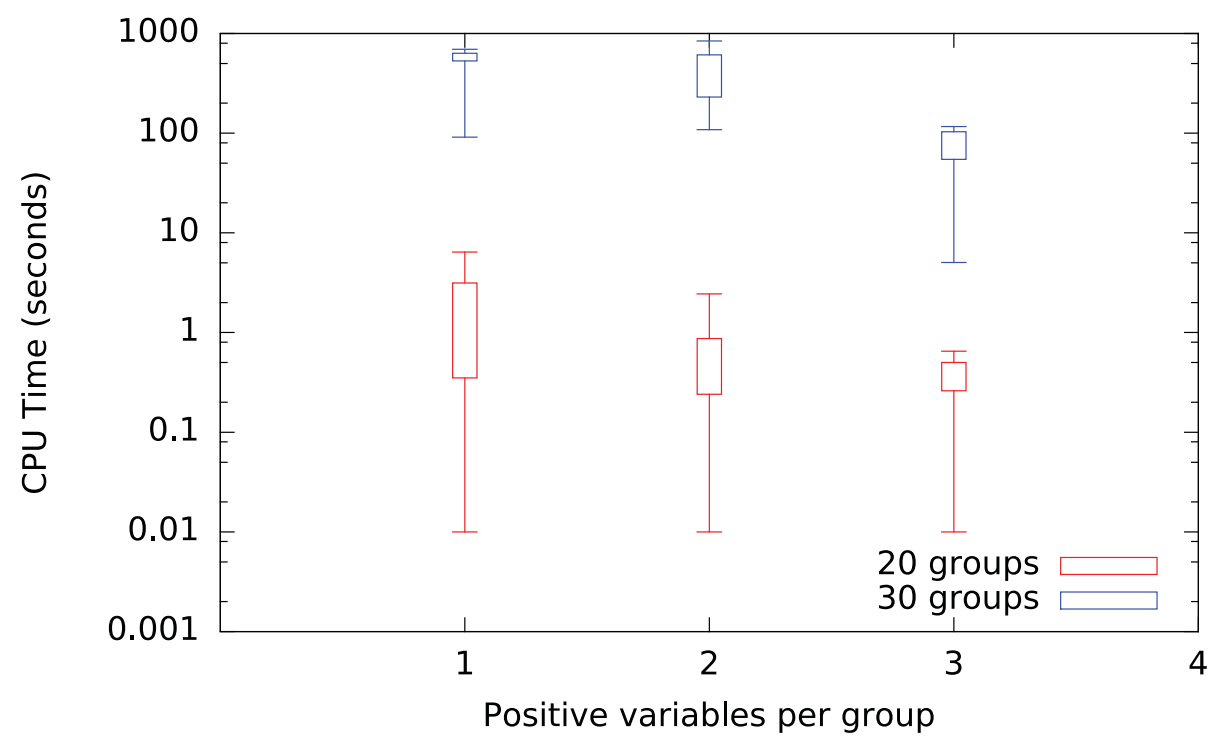

Fig. 7. Execution time against positive variables per group for a fixed number of groups.

Hard-combinatorial (crafted). These are typically the smallest benchmarks and are constructed purely in order to be difficult to solve or demonstrate some principle. The sgen 6 benchmarks were included in this category.

The benchmarks in the Application and Random categories were all longer than the 500- to 1,200-literal range of interest here, therefore only the Crafted results are considered. There was a limit of 5,000s on the CPU time permitted for any solver. The results are available on the competition Web site [Belov et al. 2014].

Figure 8 displays the results from the Hard-combinatorial SAT+UNSAT track in a form suitable for evaluating the relation $\preceq_{S C}$ where $S C$ is the set of solvers used in the competition. Each point on the plot gives the size (number of literals) of a formula and the VBS execution time for that formula over all the solvers in the competition. If a formula $f_{1}$ appears below and to the right of $f_{2}$ in Figure 8 , then $f_{1} \preceq_{S C} f_{2}$, that is, $f_{1}$ is easier than $f_{2}$. To preserve legibility, both axes use logarithmic scales and execution times of less than $0.1 \mathrm{~s}$ are recorded as 0.1 (which improves the case for formulae other than sgen6).

One additional point, labelled Extra sgen6, has been included. The smallest sgen6 formula submitted to the competition was 840 literals, thus could not be compared with the $\mathrm{Z} 1$ formulae, which were between 600 and 700 literals with minimum execution times less than $0.01 \mathrm{~s}$. We want every other formula to be comparable with at least one sgen 6 formula, therefore on our own platform we ran glueSplit_clasp (the best solver for sgen6) on a 600-literal sgen6 formula and found the execution time to be $3.93 \mathrm{~s}$. Comparison of other results suggests that execution times on our computer are approximately twice those of the competition environment, therefore we included the point (600 literals, 2s). Clearly, this value is not particularly accurate, but it is convincingly more than the $0.004 \mathrm{~s}$ of the $\mathrm{Z} 1$ formulae.

From the SAT Competition results, the solvers that give the best results for the sgen 6 formulae were glueSplit_clasp and lingeling; we thus focused our final experiments on these solvers. glueSplit_clasp incorporates clasp, therefore we included that as well. 


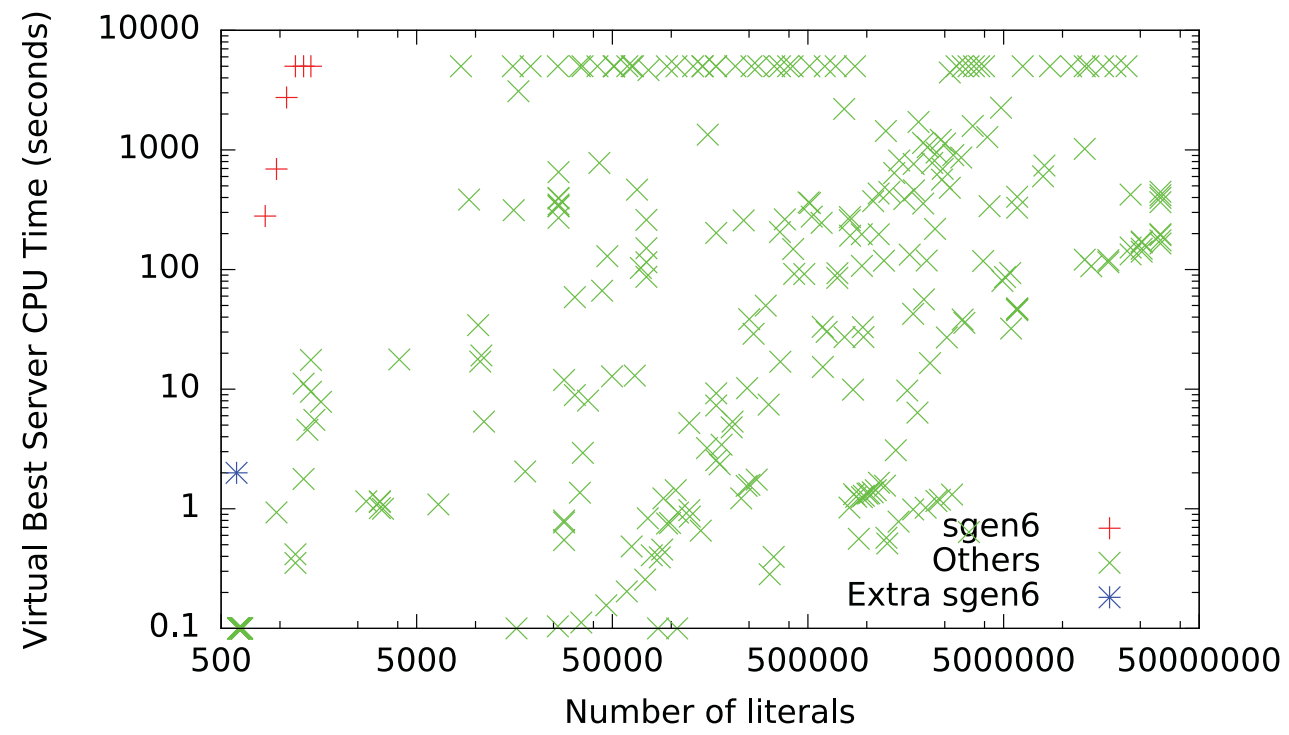

Fig. 8. Minimum times for solution of crafted benchmarks—SAT 2014.

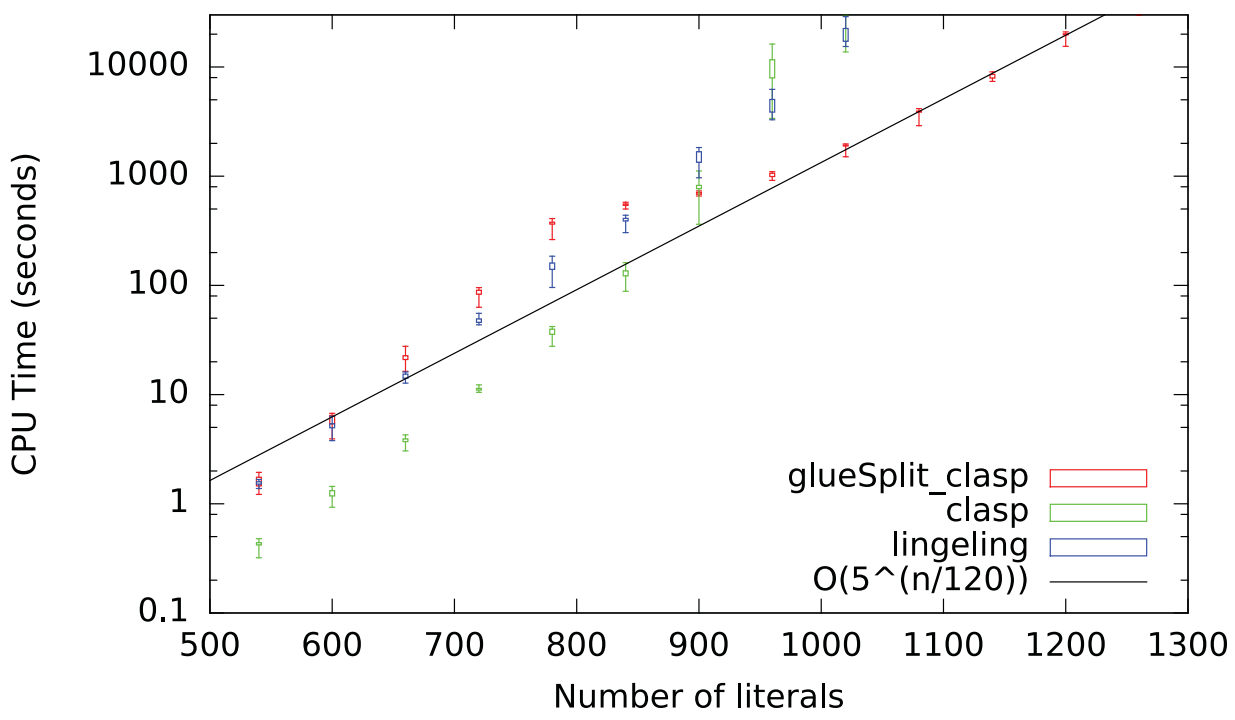

Fig. 9. Minimum execution times for sgen6 formulae.

\subsection{Further Results}

For every multiple of 60 from 540 to 1,200 literals, we generated 20 sgen 6 formulae with $g=5, p=1$, and ran each of these three solvers on the formulae with a timeout of 30,000 s. The results are shown in Figure 9. It can be seen that glueSplit_clasp gives the best results, with a complexity that appears to be of order $5^{n / 120}$.

\section{ANALYSIS}

We consider first Figure 8, which shows the Virtual Best Solver CPU time plotted against size in literals for all the entries in the Hard Combinatorial track of the SAT 
Competition 2014, together with a single added point for sgen6, as explained earlier. The largest Time value that appears corresponds to the competition timeout of $5,000 \mathrm{~s}$. On this chart, the gradient of the lining joining two points can be used to determine the relative difficulties of the corresponding formulae. If the gradient is positive, the difficulties cannot be compared, whereas if the gradient is negative, zero or infinite (vertical) then the formula appearing to the left/higher is the more difficult.

From this plot, it can be seen that there is no formula that is harder than (i.e, to left and above) any sgen 6 formula. That is, if $F_{s}$ is the set of sgen 6 formulae and $F_{o}$ is the set of all other formulae in the competition,

$$
\forall f_{s} \in F_{s}, f_{o} \in F_{o} \mid f_{s} \npreceq f_{o} .
$$

It can also be seen that for every formula that is not from sgen6, there is an sgen 6 formula that is harder than it is. That is

$$
\forall f_{o} \in F_{o} \exists f_{s} \in F_{s} \mid f_{o} \preceq f_{s} .
$$

There is a caveat to these comments, which is that if two formulae both result in a vbs timeout value, then there is not enough information to compare them (which is not the same as saying that they are incomparable). However, if only one formula results in a timeout, the comparison is valid.

Both axes of Figure 8 use logarithmic scales; it can be seen that the sgen 6 formulae are not just the most difficult but significantly so. Their vbs times for formulae of even nearly comparable size are 10 times greater, and other formulae whose vbs times are similar are nearly 10 times as long.

Figure 9 demonstrates the increase in execution time with formula size of the three best solvers for sgen6 formulae. For 1,000 literals and over the best solver is glueSplit_clasp, its complexity appears to be $O\left(5^{n / 120}\right)$, where $n$ is the number of literals. Given that the addition of a group of 5 variables increases the number of literals by 30 , this means that the addition of four groups of 5 variables multiplies the execution time by 5 .

\section{CONCLUSIONS}

On the basis of the results of the SAT 2014 competition and confirmed by our own experiments, we have demonstrated that sgen 6 generates the most difficult unsatisfiable formulae for state-of-the-art solvers. In particular, solvers that use cardinality constraint detection and are able to solve quickly what were previously regarded as the most difficult formulae cannot quickly solve sgen 6 formulae. For an unsatisfiable formula generated by sgen4, the relaxation of cardinality constraints to real-valued variables yields an unsatisfiable problem, whereas sgen6 formulae relax to problems that can be satisfied by the assignment of $1 / \mathrm{g}$ to each variable. Therefore, no solver for the real-valued relaxation, including one using linear programming, can solve these formulae. The increase in difficulty for unsatisfiable formulae is significant, with execution times for the smallest formulae at least 10 times those for other formulae of comparable length.

There remains the possibility that a new solver could be written that is targeted specifically for these formulae, but (unlike the previous situation) it is not apparent how this could be done. There are both satisfiable and unsatisfiable sgen 6 formulae with similar cardinality structures; it would appear that this is what increases the difficulty so much. 


\section{APPENDIX}

\section{A.1. Running sgen 6}

The source code sgen.c for the generator is available from the JEA library. It can be compiled with the command

gcc -o sgen sgen.c -lm

and writes the generated formula to standard output so that a typical execution would be

$$
\text { ./sgen -unsat -lits } 600>\text { sgen6-600.cnf }
$$

The arguments are:

-version integer-value.

specifies a version number that can be either 4 or 6 and defaults to 6 .

-sat | -unsat | -unknown.

indicates whether a satisfiable, unsatisfiable, or unknown formula is to be generated. A satisfiable formula is generated by constraining the permutation process as described earlier. An unsatisfiable formula is generated by testing and repeated generation using successive seeds. Exactly one of these arguments must be given for version 6 . For version 4 , unknown is not valid. -unsat is supported only if -pos-per-group is 1.

-vars integer-value.

specifies the maximum number of variables to be created. For version 6 , the number of variables will be a multiple of 5 .

-lits integer-value.

specifies the maximum number of literals to be created. For version 6 , with default values for group-size and pos-per-group, the number of variables will be a multiple of 30. Exactly one of -lits and -vars must be specified.

-group-size integer-value.

specifies the value of $g$, the size of the groups of variables. Defaults to 5 .

-pos-per-group.

specifies the value of $p$, the number of variables in each group that must be positive. Defaults to 1 .

-s integer-value.

specifies a seed for the random number generator. Repeatedly using the same seed generates the same formula. Defaults to 1 .

-model string-value.

specifies the name of a file into which a satisfying model will be written. This is applicable only if a satisfiable formula is being generated.

-min-variables.

specifies that a formula with a minimum number of variables is to be generated. -reorder.

as a final generation step, the variables and clauses are randomly permuted.

\section{ACKNOWLEDGMENTS}

The author would like to thank the organizers of the SAT Competition 2014 and the anonymous referees who made helpful comments on earlier versions of this article.

\section{REFERENCES}

Anton Belov, Daniel Diepold, Marijn Heule, and Matti Järvisalo. 2014. SAT Competition Website. Retrieved April 16, 2015 from http://www.satcompetition.org/2014/.

Armin Biere, Daniel Le Berre, Emmanuel Lonca, and Norbert Manthey. 2014. Detecting cardinality constraints in cnf. In Theory and Applications of Satisfiability Testing SAT 2014, Carsten Sinz 
and Uwe Egly (Eds.). Lecture Notes in Computer Science, Vol. 8561. Springer, Berlin, $285-301$. DOI : http://dx.doi.org/10.1007/978-3-319-09284-3_22

Stephen A. Cook. 1971. The complexity of theorem-proving procedures. In STOC'71: Proceedings of the 3rd Annual ACM Symposium on Theory of Computing. ACM, New York, NY, 151-158. DOI : http://dx.doi.org/ $10.1145 / 800157.805047$

W. Cook, C. R. Coullard, and G. Turn. 1987. On the complexity of cutting-plane proofs. Discrete Applied Mathematics 18, 1, 25-38. DOI : http://dx.doi.org/10.1016/0166-218X(87)90039-4

DIMACS. 1993. Satisfiability Suggested Format. www.satlib.org/Benchmarks/SAT/satfomat.ps, accessed 7th May 2015.

Armin Haken. 1985. The intractability of resolution. Theoretical Computer Science 39, 297-308.

Edward Hirsch. 2002. Random Generator hgen2 of Satisfiable Formulas in 3-CNF. Retrieved from http://logic. pdmi.ras.ru/hirsch/benchmarks/.

Matti Järvisalo, Daniel Le Berre, Olivier Roussel, and Laurent Simon. 2012. The international SAT solver competitions. AI Magazine 33, 1, 89-92.

$\mathrm{Chu}$ Min Li. 2000. Integrating equivalency reasoning into Davis-Putnam procedure. In Proceedings of the 17th National Conference on Artificial Intelligence (AAAI'00). 291-296.

Mladen Mikša and Jakob Nordström. 2014. Long proofs of (seemingly) simple formulas. In Theory and Applications of Satisfiability Testing SAT 2014, Carsten Sinz and Uwe Egly (Eds.). Lecture Notes in Computer Science, Vol. 8561. Springer, 121-137. DOI : http://dx.doi.org/10.1007/978-3-319-09284-3_10

Richard Ostrowski, Éric Grégoire, Bertrand Mazure, and Lakhdar Sais. 2002. Recovering and exploiting structural knowledge from CNF formulas. In Principles and Practice of Constraint Programming (CP'02), Pascal Van Hentenryck (Ed.). 185-199.

Ivor Spence. 2010. Sgen1: A generator of small but difficult satisfiability benchmarks. ACM Journal of Experimental Algorithmics 15. DOI : http://dx.doi.org/10.1145/1671970.1671972

Allen Van Gelder and Ivor Spence. 2010. Zero-One designs produce small hard SAT instances. In Theory and Applications of Satisfiability Testing SAT 2010, Ofer Strichman and Stefan Szeider (Eds.). Lecture Notes in Computer Science, Vol. 6175. Springer, Berlin, 388-397. D0I:http://dx.doi. org/10.1007/978-3-642-14186-7_37

Joost P. Warners and Hans Van Maaren. 1998. A two phase algorithm for solving a class of hard satisfiability problems. Operations Research Letters 23, 81-88.

J. Eldon Whitesitt. 1995. Boolean Algebra and Its Applications. Dover Publications, Mineola, NY.

Lin Xu, Frank Hutter, Holger Hoos, and Kevin Leyton-Brown. 2012. Evaluating component solver contributions to portfolio-based algorithm selectors. In Theory and Applications of Satisfiability Testing SAT 2012, Alessandro Cimatti and Roberto Sebastiani (Eds.). Lecture Notes in Computer Science, Vol. 7317. Springer, Berlin, 228-241. DOI : http://dx.doi.org/10.1007/978-3-642-31612-8_18

Received September 2014; revised February 2015; accepted March 2015 